\title{
Four Swift searches for transient sources of high-energy neutrinos
}

\author{
A. Keivani*, D. F. Cowen, D. B. Fox, J. A. Kennea, G. Tešić, and C. F. Turley \\ Pennsylvania State University, University Park, PA 16802, USA \\ E-mails: keivani@psu.edu, dfc13epsu.edu, dfox@astro.psu.edu, \\ kennea@swift.psu.edu, gordana@tesic.net, cft114@psu.edu
}

\author{
P. A. Evans, J. P. Osborne \\ University of Leicester, Leicester, LEI 7RH, UK \\ E-mail: pae9@leicester.ac.uk, opj@leicester.ac.uk
}

\section{F. E. Marshall}

NASA Goddard Space Flight Center, Mail Code 661, Greenbelt, MD 20771, USA

E-mail: frank.marshall@nasa.gov

\begin{abstract}
We present results of the first four Swift satellite follow-up campaigns seeking to identify transient or variable X-ray or UV/optical sources that might be associated with individual candidate highenergy cosmic muon neutrinos detected by the IceCube Neutrino Observatory. Real-time public alerts providing coordinates and arrival times of likely-cosmic neutrinos have been provided by IceCube, via the Astrophysical Multimessenger Observatory Network, since April 2016. Subsequent Swift X-ray observations of four likely-cosmic neutrinos (events 160731A, 161103A, $170312 \mathrm{~A}$, and 170321A) reveal multiple X-ray sources in the targeted 90\%-containment regions, most of which have been previously identified, and none of which are considered likely sources of high-energy neutrinos. Observations exclude association with the brightest $30 \%$ to $65 \%$ of Swifttype $\gamma$-ray burst X-ray afterglows over the observed regions. Contemporaneous Swift UV/optical observations, providing reduced coverage of the event localizations, also reveal no candidate transient or variable UV/optical counterparts. We discuss the results of these campaigns and our plans for further follow-up of likely-cosmic high-energy neutrinos from IceCube.
\end{abstract}

35th International Cosmic Ray Conference - ICRC2017

10-20 July, 2017

Bexco, Busan, Korea

${ }^{*}$ Speaker. 


\section{Introduction}

The detection of high-energy ( $E_{v} \gtrsim 1 \mathrm{TeV}$ ) astrophysical neutrinos has been reported, and confirmed with over five years of data, by IceCube [1]. However, the nature of the cosmic sources of these neutrinos remains unknown. If these sources are transient in nature, they might be accompanied by bright electromagnetic (EM) counterparts that could be identified in rapid-response target of opportunity (TOO) observations at X-ray and other wavelengths. The Astrophysical Multimessenger Observatory Network (AMON) ${ }^{1}[2,3]$ was founded in part to search for and study such "multimessenger" transient sources. Candidate source populations include $\gamma$-ray bursts (GRBs); blazars and other types of active galactic nuclei (AGN); ultra-luminous star-forming galaxies (nontransient); and low-luminosity GRBs or other types of supernova (for a recent review, see [4]).

Given AMON and community interest in rapid-response follow-up observations, IceCube and AMON collaborated to implement real-time analysis and triggering of individual likely-cosmic high-energy neutrino events. To date two Gamma-ray Coordinates Network (GCN) [5] event streams have been commissioned: the AMON_ICECUBE_HESE "High Energy Starting Event" (HESE) track-like event stream in April 2016, and the AMON_ICECUBE_EHE "Extremely High Energy" (EHE) event stream in July 2016.

Working from these public alert streams, we have carried out four searches for luminous EM counterparts to HESE and EHE events with NASA's Swift satellite [6], focusing on data from its X-ray Telescope (XRT) [7] and UV/Optical Telescope (UVOT) [8]. The goal of these observations is to either identify the first EM counterparts to high-energy cosmic neutrinos or to set useful constraints on the nature of any associated transient or flaring emissions. An unusual source appearing in the localization region with appropriate timing, energy, and spectral properties, supporting a scenario for joint high-energy neutrino emission, could reasonably be claimed as the first EM counterpart to any high-energy neutrino.

Our paper proceeds as follows: Section 2 reviews the properties of the four targeted candidate cosmic neutrinos, along with our Swift follow-up observations. Section 3 derives quantitative results for the GRB source scenario. We conclude in Section 4.

\section{Observations}

From April 2016 through May 2017, the IceCube online system [9] identified six HESE and four EHE neutrinos that were distributed publicly in real-time through AMON and GCN [10]. One of these neutrinos, IceCube-160731A, triggered both HESE and EHE streams. We triggered Swift follow-up observations of four of these alerts: IceCube-160731A, IceCube-161103A, IceCube170312A, and IceCube-170321A.

\subsection{IceCube Data}

IceCube currently distributes two distinct event streams via AMON, for HESE and EHE tracklike events [10]. Key parameters reported for both streams are the arrival direction of the event

\footnotetext{
${ }^{1}$ AMON website: http://sites.psu.edu/amon/
} 
(R.A. and Dec.), localization uncertainty ( $r_{50}$ for $50 \%$-containment; $r_{90}$ for $90 \%$-containment), arrival time, and revision number. Any refined localizations are distributed via GCN as alert revisions, with revision number incremented by one at each instance.

The AMON_ICECUBE_HESE stream includes two additional parameters for each event. The charge parameter reports the causally-connected charge, with higher-charge events more likely to have an astrophysical origin. The signal_trackness parameter, a number between zero and one, reflects the likelihood that the individual HESE neutrino is both signal-like (astrophysical) and track-like.

The AMON_ICECUBE_EHE stream includes an estimate of the deposited charge, an estimate of the neutrino energy, and the parameter signalness, an estimate of the probability that the event was due to an astrophysical (rather than atmospheric) neutrino.

IceCube-160731A - This event was detected at 01:55:04 UT on 31 July 2016 with charge = 15814.74 photoelectrons and signal_trackness $=0.91$. This event was identified as a HESE neutrino. The initial localization of this event was reported as $0.42^{\circ}, 50 \%$ error radius $\left(1.23^{\circ}, 90 \%\right.$ error radius $)$ around position RA, Dec $(\mathrm{J} 2000)=\left(215.11^{\circ},-0.46^{\circ}\right) 41$ seconds after detection i.e. at 01:55:45 UT as Rev. 0. IceCube later updated the localization $0.35^{\circ}, 50 \%$ error radius $\left(0.75^{\circ}, 90 \%\right.$ error radius) around position RA, Dec $(\mathrm{J} 2000)=\left(214.54^{\circ},-0.33^{\circ}\right)$ at 02:35:38 UT on 1 August 2016 (Rev. 1). This event also triggered the EHE stream at 01:55:58 UT. Triggering both pipelines is a rare occurrence, anticipated to happen roughly once per 5 years. The initial localization of this event was reported as $0.17^{\circ}, 50 \%$ error radius around position RA, Dec $(\mathrm{J} 2000)=\left(215.09^{\circ}\right.$, $\left.-0.42^{\circ}\right), 54$ seconds after detection as Rev. 0 . IceCube later updated the position $\left(214.54^{\circ},-0.33^{\circ}\right)$ at 02:35:54 UT on August 1, 2016 as Rev. 1.

IceCube-161103A - This event was detected at 09:07:31 UT on 3 November 2016 with charge $=$ 7546.05 photoelectrons and signal_trackness $=0.30$. This event was identified as a HESE neutrino. The initial localization of this event was reported as $0.42^{\circ}, 50 \%$ error radius $\left(1.23^{\circ}, 90 \%\right.$ error radius $)$ around position RA, Dec $(\mathrm{J} 2000)=\left(40.87^{\circ},+12.62^{\circ}\right) 40$ seconds after detection i.e. at 09:08:11 UT as Rev. 0. IceCube later updated the localization $0.65^{\circ}, 50 \%$ error radius $\left(1.1^{\circ}\right.$, $90 \%$ error radius) around position $\left(40.82^{\circ},+12.56^{\circ}\right)$ at 14:07:40 UT on the same day (Rev. 1).

IceCube-170312A - This event was detected at 13:49:39 UT on 12 March 2017 with charge = 8858.64 photoelectrons and signal_trackness $=0.78$. This event was identified as a HESE neutrino. The initial localization of this event was reported as $0.42^{\circ}, 50 \%$ error radius $\left(1.23^{\circ}, 90 \%\right.$ error radius $)$ around position RA, Dec $(\mathrm{J} 2000)=\left(304.73^{\circ},-26.24^{\circ}\right) 50$ seconds after detection i.e. at 13:50:29 UT as Rev. 0. IceCube later updated the localization $<0.5^{\circ}, 90 \%$ error radius around position $\left(305.15^{\circ},-26.61^{\circ}\right)$ at 02:29:21 UT on March 13, 2017 (Rev. 1).

IceCube-170321A - This event was detected at 07:32:20 UT on 21 March 2017 with charge $=$ 6214.41 photoelectrons and signalness $=0.28$. This event was identified as an EHE neutrino. The initial localization of this event was reported as $0.32^{\circ}, 50 \%$ error radius around position RA, Dec $(\mathrm{J} 2000)=\left(98.33^{\circ},-14.49^{\circ}\right) 38$ seconds after detection i.e. at 07:32:58 UT as Rev. 0. IceCube later updated the localization $1.2^{\circ}, 90 \%$ error radius around position $\left(98.30^{\circ},-15.02^{\circ}\right)$ at $01: 02: 06$ UT on March 22, 2017 (Rev. 1). 


\subsection{Swift Data}

Swift carried out rapid follow-up observations for the four IceCube events listed above. These were the first real-time searches for high-energy neutrino counterparts with Swift which were a Priority 1 TOO mosaic of 19 (or 7 for the last event) Swift pointings under our Swift Cycle 12 Guest Investigator program. No transient sources were discovered in any of these searches associated with the IceCube trigger $[11,12,13,14]$. Automated analysis of the XRT data was carried out as the data are received using the automated reduction routines described in [15].

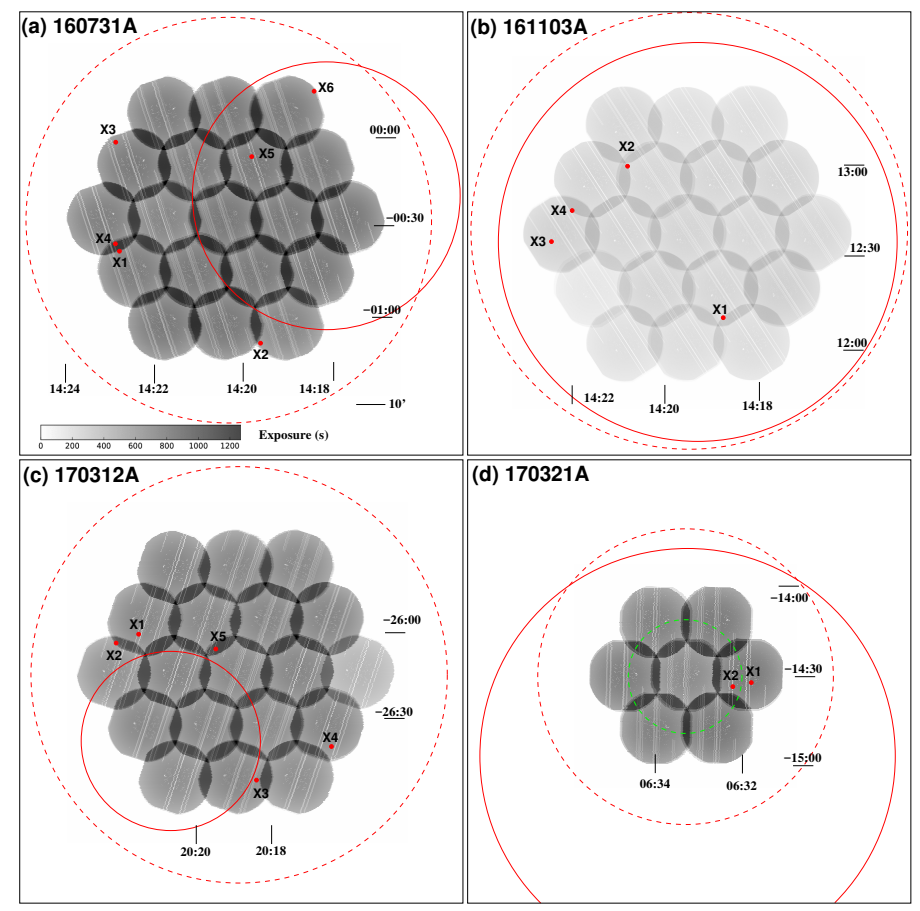

Figure 1: Swift XRT follow-up campaigns for (a) IceCube-160731A, (b) IceCube-161103A, (c) IceCube-170312A, and (d) IceCube-170321A. Each panel shows the X-ray exposure map resulting from the adopted 19- or 7-point tiling pattern centered on the initial IceCube neutrino localization (greyscale), and the positions of all detected X-ray sources (red points). Red dashed circles show initial 90\%-containment regions, and red solid circles the final 90\%-containment regions, for each neutrino. Only a 50\%-containment radius was reported in Rev. 0 for IceCube-170321A (green dashed circle in $(d)$ ). Color bar represents achieved exposure at each sky position.

IceCube-160731A - Swift followed up IceCube-160731A within an hour of neutrino arrival time at the South Pole (01:55:04 UT, July 31, 2016). The HESE alert of IceCube-160731A arrived earlier than the EHE, so the Swift automated system first triggered the HESE alert and performed followup observations centered on its initial position. The observations were taken between 03:00:46 and 14:51:52 UT on July 31, 2016 and covered $2.1 \mathrm{deg}^{2}$. Swift covered a region centered on RA, $\operatorname{Dec}(\mathrm{J} 2000)=\left(215.11^{\circ},-0.46^{\circ}\right)$, with a radius of approximately $0.8^{\circ}$. This covers $64.2 \%$ of the neutrino revised $r_{90}$ error region. Swift XRT collected $\approx 800 \mathrm{~s}$ per field of PC mode data per tile.

Six X-ray sources were detected in the observations, with all six corresponding to known $\mathrm{X}$-ray emitters or catalog objects from which persistent X-ray emission may be expected. We 
did not discover any transient X-ray event associated with the IceCube trigger. Details of the six detected X-ray sources are discussed in our GCN circular [11]. The $3 \sigma$ upper limit on the count rate in the rest of the field was $0.01 \mathrm{ct} \mathrm{s}^{-1}$, which corresponded to a $0.3-10 \mathrm{keV}$ flux of $4.3 \times$ $10^{-13} \mathrm{erg} \mathrm{cm}^{-2} \mathrm{~s}^{-1}$ for a typical AGN spectrum $\left(N_{\mathrm{H}}=3 \times 10^{20} \mathrm{~cm}^{-2}, \Gamma=1.7\right)$. Overlaps between the different tiles accounted for $0.5 \mathrm{deg}^{2}$ : in these regions the $3 \sigma$ upper limit was $0.007 \mathrm{ct} \mathrm{s}^{-1}$, corresponding to $3.1 \times 10^{-13} \mathrm{erg} \mathrm{cm}^{-2} \mathrm{~s}^{-1}$. Fig. 1(a) shows the 19-point tiling pattern centered on the IceCube neutrino, as the Swift exposure map, and the six detected X-ray sources.

No plausible counterparts are found in the UVOT data. All 19 tiling positions were observed using the $U$ filter. Each position was typically observed 4 times with a total exposure of about $420 \mathrm{~s}$; the longest individual exposure for a position was about $120 \mathrm{~s}$. The limiting sensitivity is about 18.9 mag. This accounts for the sensitivity of UVOT and the depth of the USNO catalog [18] used to reject known sources.

IceCube-161103A - Swift followed up IceCube-161103A within about five hours of neutrino arrival time at the South Pole (09:08:11 UT, November 3, 2016). The main reason for this delay was that pointing the XRT at the neutrino direction would have resulted in the radiator pointing towards the Sun so immediate observing would have overheated the XRT. The observations were taken between 13:58:30 UT and 18:55:15 UT on November 3, 2016 and covered $2.1 \mathrm{deg}^{2}$. Swift covered a region centered on RA, Dec $(\mathrm{J} 2000)=\left(40.87^{\circ},+12.62^{\circ}\right)$, with a radius of approximately $0.8^{\circ}$. This covers $68.0 \%$ of the neutrino revised $r_{90}$ error region. Swift XRT collected between $150 \mathrm{~s}$ and $250 \mathrm{~s}$ of PC mode data per tile.

Four X-ray sources were detected in the observations. None of these are known X-ray emitters, however all are faint, and well below the ROSAT All-Sky Survey (RASS) limits [17], therefore we do not consider any of them likely counterparts to the IceCube trigger. Details of the four detected X-ray sources are discussed in our GCN circular [12]. The $3 \sigma$ upper limit on the count rate in the rest of the field was $0.03 \mathrm{ct} \mathrm{s}^{-1}$, which corresponds to a $0.3-10 \mathrm{keV}$ flux of $1.2 \times 10^{-12} \mathrm{erg} \mathrm{cm}^{-2} \mathrm{~s}^{-1}$. Overlaps between the different tiles accounts for $0.5 \mathrm{deg}^{2}$ : in these regions the $3 \sigma$ upper limit is $0.02 \mathrm{ct} \mathrm{s}^{-1}$, corresponding to $8.1 \times 10^{-13} \mathrm{erg} \mathrm{cm}^{-2} \mathrm{~s}^{-1}$. Fig. 1(b) shows the 19-point tiling pattern centered on the IceCube neutrino, as the Swift exposure map, and the four detected X-ray sources.

No plausible counterparts are found in the UVOT data. UVOT also observed 19 different pointing directions, with the $U$ filter used for 16 pointings and the UVW1 filter used for the rest. The typical observing exposure was about $250 \mathrm{~s}$ with multiple visits. The limiting sensitivity is about $U=18.9$ mag.

IceCube-170312A - Swift followed up IceCube-170312A about two hours after neutrino arrival time at the South Pole (13:49:39 UT, March 12, 2017). The observations were taken between 15:51:22 UT on March 12, 2017 and 05:04:48 UT on March 13, 2017 (i.e. from 7.3 ks to $54.9 \mathrm{ks}$ after the neutrino trigger), Swift covered a region centered on RA, Dec $(\mathrm{J} 2000)=\left(304.73^{\circ},-26.24^{\circ}\right)$, with a radius of approximately $0.8^{\circ}$. This covers $82.3 \%$ of the neutrino revised $r_{90}$ error region. Swift XRT collected $\sim 800$ s of PC mode data per tile.

Five X-ray sources were detected in the observations. One of these corresponds to a known $\mathrm{X}$-ray emitter, and others are faint objects below the RASS limits [17], therefore we do not consider any of them to be likely counterparts to the IceCube trigger. Details of the four detected X-ray 
sources are discussed in our GCN circular [13]. The $3 \sigma$ upper limit on the count rate in the rest of the field was $0.01 \mathrm{ct} \mathrm{s}^{-1}$, which corresponds to a $0.3-10 \mathrm{keV}$ flux of $4.1 \times 10^{-13} \mathrm{erg} \mathrm{cm}^{-2} \mathrm{~s}^{-1}$. Overlaps between the different tiles accounts for $0.5 \mathrm{deg}^{2}$ : in these regions the $3 \sigma$ upper limit is $0.007 \mathrm{ct} \mathrm{s}^{-1}$, corresponding to $2.9 \times 10^{-13} \mathrm{erg} \mathrm{cm}^{-2} \mathrm{~s}^{-1}$. Fig. 1(c) shows the 19-point tiling pattern centered on the IceCube neutrino, as the Swift exposure map, and the five detected X-ray sources.

No plausible counterparts are found in the UVOT data. All 19 tiling positions were observed using the $U$ filter. The typical observing exposure was about $110 \mathrm{~s}$ with a single visit. The limiting sensitivity is about $U=18.9$ mag.

IceCube-170321A - Swift followed up IceCube-170321A about six hours after neutrino arrival time at the South Pole (07:32:20 UT, March 21, 2017). The observations were taken between 14:09:02 UT and 18:03:00 UT on March 21, 2017 and covered $0.77 \mathrm{deg}^{2}$. Swift covered a region centered on RA, Dec $(\mathrm{J} 2000)=\left(98.33^{\circ},-14.49^{\circ}\right)$, with a radius of approximately $0.55^{\circ}$. This covers $22.1 \%$ of the neutrino revised $r_{90}$ error region. Swift XRT collected $\sim 900 \mathrm{~s}$ of PC mode data per tile.

Two X-ray sources were detected in the observations. Both of these correspond to known $\mathrm{X}$-ray emitters. We did not discover any transient X-ray event associated with the IceCube trigger. Details of the brighter X-ray source are reported in our GCN circular [14]. The $3 \sigma$ upper limit on the count rate of any such counterpart is $0.004 \mathrm{ct} \mathrm{s}^{-1}$, which corresponds to a $0.3-10 \mathrm{keV}$ flux of $1.48 \times 10^{-13} \mathrm{erg} \mathrm{cm}^{-2} \mathrm{~s}^{-1}$. Fig. 1(d) shows the 7-point tiling pattern centered on the IceCube neutrino, as the Swift exposure map, and the two detected X-ray sources.

No plausible counterparts are found in the UVOT data. All 7 tiling positions were observed using the $U$ filter. The mean observing exposure was $922 \mathrm{~s}$ with multiple visits. The limiting sensitivity is about $U=18.9$ mag.

\section{Discussion}

To evaluate constraints on any associated GRB X-ray afterglows, we use a library of 192 Swift XRT lightcurves and piecewise power-law fits for Swift-detected GRB afterglows [19]. Using power-law fits allows us to calculate fluxes for these afterglows at arbitrary times; since fits exclude intervals of X-ray flaring (e.g. [20]) this approach is conservative with respect to such activity.

Figure 2 shows the median X-ray afterglow flux (black line), and 80\%-confidence (light grey) and 50\%-confidence (dark grey) ranges on afterglow flux, over the period from $t+4 \mathrm{ks}$ to $t+60 \mathrm{ks}$ post-burst relevant to our observations (assuming the neutrino detection time to be coincident with the GRB). Superposed on the afterglow flux ranges are X-ray flux limits for the IceCube-160731A (orange), IceCube-161103A (red), IceCube-170312A (cyan), and IceCube-170321A (yellow) campaigns, averaged over the 7 or 19 tiles of each mosaic pointing. These limits are derived from the exposure history of these observations using Poisson statistics and a standard XRT afterglow counts to flux conversion factor of $4 \times 10^{-11} \mathrm{erg} \mathrm{cm}^{-2} \mathrm{ct}^{-1}$. Specifically, the flux limit is defined as the number of source photons required (at any given time) to yield an excess over background with p-value $p \leq 10^{-6}$ in a single source aperture. Such excesses will occur via Poisson fluctuation of the background in roughly $10 \%(4 \%)$ of 19-tile (7-tile) observing campaigns. Our limits indicate that 65\% (IceCube-160731A), 30\% (IceCube-161103A), 55\% (IceCube-170312A), and 43\% 


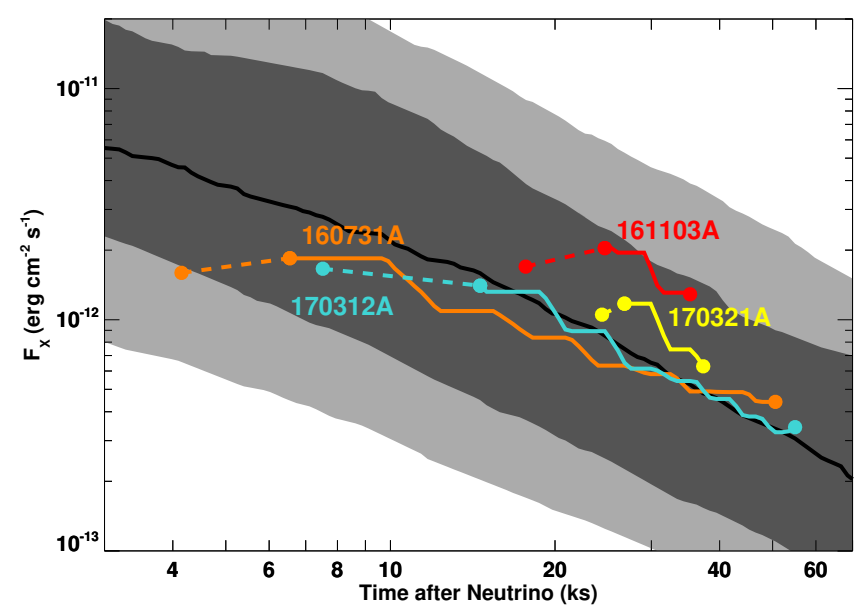

Figure 2: X-ray flux limits for the Swift XRT follow-up campaigns on IceCube-160731 A (orange), IceCube-161103A (red), IceCube-170312A (cyan), and IceCube-170321A (yellow) along with the median flux (black line) and 80\%-confidence (light grey) and 50\%-confidence (dark grey) ranges on afterglow flux for the library of 192 Swift XRT afterglow light curves (and piecewise power-law fits) from [19]. Solid lines show limits from completion of the first observation of the last pointing until the end of the campaign, while dashed lines show the initial intervals of partial coverage.

(IceCube-170321A) of the X-ray afterglows of Swift-detected GRBs would be recovered by the follow-up campaigns of these neutrinos, assuming the burst occurred within the field of view of the observations, and ignoring the increased sensitivity provided over regions of pointing overlap.

\section{Conclusion}

We have reported the results of the first four Swift satellite follow-up campaigns on high-energy neutrino alerts from the IceCube neutrino observatory, IceCube-160731A, IceCube-161103A, IceCube170312A, and IceCube-170321A. In observations covering 64.2\%, 68.0\%, 82.3\%, and $22.1 \%$ of the $90 \%$ containment regions for IceCube-160731A, IceCube-161103A, IceCube-170312A, and IceCube-170321A, with mean epochs of $t+13 \mathrm{~h}, t+10 \mathrm{~h}, t+15 \mathrm{~h}$, and $t+10 \mathrm{~h}$ post-neutrino, we identify no compelling candidate X-ray or UV/optical counterpart for any of the events. We place $3 \sigma$ upper limits on the flux for a typical AGN spectrum.

We discussed these limits in the context of Swift-detected gamma-ray bursts (GRBs). Our limits indicate that $30 \%-65 \%$ of the X-ray afterglows of Swift-detected GRBs would be recovered by the follow-up campaigns of these neutrinos. We plan to continue Swift follow-up observations of IceCube high-energy neutrinos at an expected rate of roughly four campaigns per year.

Acknowledgements. The authors thank the IceCube collaboration for publicly distributing highenergy neutrinos in real-time, and the Swift team for their rapid responses to these alerts. A.K., D.B.F., D.F.C., G.T. and C.F.T. acknowledge support from the National Science Foundation under grant PHY-1412633; A.K. and D.F.C. acknowledge support from the National Aeronautics and Space Administration Swift Guest Investigator Program under grant NNX17AI95G. A.K. and G.T. 
gratefully acknowledge support from the Institute for Gravitation and the Cosmos at the Pennsylvania State University. P.A.E. and J.P.O. acknowledge support from the UK Space Agency.

\section{References}

[1] IceCube Collaboration, Observation of Astrophysical Neutrinos in Six Years of IceCube Data, in proceeding of 35th ICRC, POS ( ICRC2017) 981 (2017)

[2] A. Keivani et al., Astrophysical Multimessenger Observatory Network (AMON): Science, Infrastructure, and Status, in proceeding of 35th ICRC, POS (ICRC2017) 629 (2017)

[3] M. W. E. Smith et al., The Astrophysical Multimessenger Observatory Network (AMON), Astroparticle Physics 45, 56-70 (2013) [arXiv: 1211 . 5602]

[4] K. Murase and E. Waxman, Constraining high-energy cosmic neutrino sources: Implications and prospects, Physical Review D, 94, 103006 (2016) [arXiv: 1607 .01601]

[5] S. D. Barthelmy et al., BACODINE, the Real-Time BATSE Gamma-Ray Burst Coordinates Distribution Network, Astrophysics and Space Science, 231, 235-238 (1995)

[6] N. Gehrels et al., The Swift Gamma-Ray Burst Mission, Astrophysical Journal, 611, 1005-1020 (2004) [arXiv:astro-ph/0405233]

[7] D. N. Burrows et al., The Swift X-Ray Telescope, Space Science Reviews, 120, 165-195 (2005) [arXiv:astro-ph/0508071]

[8] P. W. A Roming et al., The Swift UV/Optical Telescope, Space Science Reviews, 120, 95-142 (2005) [arXiv:astro-ph/0507413]

[9] M. G. Aarsten et al., The IceCube realtime alert system, Astroparticle Physics, 92, 30-41 (2017) [arXiv:1612.06028]

[10] GCN/AMON NOTICE, https://gcn.gsfc.nasa.gov/amon.html

[11] P. A. Evans et al., https://gcn.gsfc.nasa.gov/gcn3/19747.gcn3

[12] P. A. Evans et al., https://gcn.gsfc.nasa.gov/gcn3/20125.gcn3

[13] A. Keivani et al., https://gcn.gsfc.nasa.gov/gcn3/20890.gcn3

[14] A. Keivani et al., https://gcn.gsfc.nasa.gov/gen3/20964.gcn3

[15] P. A. Evans et al., Methods and results of an automatic analysis of a complete sample of Swift-XRT observations of GRBs, MNRAS, 397, 1177-1201 (2009) [arXiv: 0812.3662]

[16] T. Abbey et al., Micrometeroid Damage to CCDs in XMM-Newton and Swift and its Significance for Future X-ray Missions, in proceeding of The X-ray Universe 2005, ESA Spec. Pub. 604, 943 (2006)

[17] W. Voges et al., The ROSAT all-sky survey bright source catalogue, Astronomy and Astrophysics, 349, 389-405 (1999) [arXiv: astro-ph/9909315]

[18] D. G. Monet et al., The USNO-B Catalog, The Astronomical Journal, 125, 984-993 (2003) [arXiv:astro-ph/0210694]

[19] J. L. Racusin et al., Jet Breaks and Energetics of Swift Gamma-Ray Burst X-Ray Afterglows, Astrophysical Journal, 698, 43-74 (2009) [arXiv: 0812 .4780]

[20] G. Chincarini et al., The First Survey of X-Ray Flares from Gamma-Ray Bursts Observed by Swift: Temporal Properties and Morphology, Astrophysical Journal, 671, 1903-1920 (2007) [arXiv:astro-ph/0702371] 